\title{
Lectin Reactivities as Intermediate Biomarkers in Premalignant Colorectal Epithelium
}

\author{
C. Richard Boland, Maria A. Martin, and Irwin J. Goldstein \\ Departments of Internal Medicine and Biological Chemistry, University of Michigan School of Medicine and \\ Veterans Affairs Medical Center, Ann Arbor, Michigan 48109
}

\begin{abstract}
Normal colonic epithelial cells undergo maturation as they traverse the crypt to the lumenal surface. The binding of lectins to goblet cell mucins and other glycoconjugates changes as the cells migrate and differentiate. Additional stepwise modifications in glycoconjugate expression occur in premalignant and malignant neoplasms that may be detected by lectin binding studies. The lectins Dolichos biflorus agglutinin (DBA) and soybean agglutinin (SBA) have been developed as markers of differentiation in normal-appearing colonic epithelium. Using a quantitative biometric system to score tissues, reduced levels of lectin binding have been found in rectal tissue from patients with familial adenomatous polyposis (FAP) and hereditary nonpolyposis colorectal cancer. The lectin Amaranthus caudatus agglutinin (ACA) binds to a cytoplasmic glycoconjugate expressed at the base of the colonic crypt and serves as a possible proliferation marker in the distal, but not proximal, colon. ACA binding increases in tandem with increased levels of proliferation (Using BrdU incorporation) in neoplastic tissues. Binding by the peanut lectin (PNA) occurs late in the adenoma-to-carcinoma sequence - in larger adenomas and in cancers - and serves as a marker of advancing neoplasia. Lectins identify the stepwise changes that occur during normal differentiation, proliferation and in advancing neoplasia. By selecting the appropriate probe, biomarkers may be developed for early, intermediate, and late events in colorectal cancer. 01992 Wiley-Liss, inc.
\end{abstract}

Key words: BrdU, cellular differentiation, chemoprevention, glycoconjugate expression, intermediate biomarker, lectins (DBA, SBA, ACA, PNA), malignant transformation, premalignant epithelium, proliferation, tumor markers

Intermediate biomarkers of neoplastic progression are an important goal in the study of colorectal cancer. Optimal biomarkers would be easy to obtain, would provide a means to measure increased risk for the development of cancer, and would be modified in a positive direction upon the introduction of appropriate changes in lifestyle or introduction of effective chemopreventive agents. Most of the previously developed intermediate biomarkers require the procurement of fresh tissue with stringent requirements on handling and processing, which can limit the acquisition of data. This communication will review the use of lectins as histochemical probes of glycoprotein biomarkers that are expressed in colorectal tissues. Lectins have been identified that are useful as markers of proliferation, differentiation, and malignant transformation in the human colon. Other investigators have emphasized that proliferation, differ- entiation, and transformation are related but separate processes, and in all likelihood no single probe is capable of providing insight into all three aspects of cellular behavior. Panels of lectins may be selected that can be used to analyze these three behaviors in the colon, and importantly, these probes may be used on fixed, paraffin-embedded tissue sections, regardless of the duration of their storage.

\section{PROLIFERATION, DIFFERENTIATION, AND MALIGNANT TRANSFORMATION IN THE COLON}

\section{Proliferation}

Cellular proliferation occurs in the lower portion of the normal colonic crypt. After cell division, one cell becomes a daughter cell 
which then migrates towards the luminal surface [1]. Proliferation has traditionally been estimated by the incorporation of DNA precursors, such as $3 \mathrm{H}$-thymidine followed by autoradiography or the incorporation of bromodeoxyuridine (BrdU) followed by immunohistochemistry [2]. Other groups have measured the presence of proliferationassociated proteins using the monoclonal antibody Ki-67 or antibodies to the proliferating cell nuclear antigen (PCNA). Additional approaches have involved measurement of the activity of ornithine decarboxylase (ODC) which is required for the generation of polyamines and proliferation [3].

\section{Differentiation}

Differentiation has been indirectly measured using $3 \mathrm{H}$-thymidine or $\mathrm{BrdU}$ incorporation. This permits an estimation of the total labeling index as well as inappropriate expansion or shift of the proliferating pool into the upper portions of the crypt [2]. However, both of these approaches require that fresh tissue be incubated with DNA precursors immediately after it is obtained. This obviates the possibility of examining material obtained previously, and requires that the tissue be obtained in conjunction with a tissue culture facility. Moreover, these methods do not measure differentiation directly; rather, they measure the failure to repress DNA synthesis.

Newly produced epithelial cells differentiate as they migrate from the base of the colonic crypt to the surface. Although hyperproliferation often occurs in the setting of impaired cellular differentiation, these two processes are not always linked to one another. Relatively few markers of epithelial cell differentiation have ever been developed for the human colon [4].

\section{Malignant Transformation}

Hyperproliferation is a characteristic of neoplastic lesions, and it has been observed that this phenomenon occurs in certain premalignant lesions as well [3]. Although the changes that occur in the normal-appearing colonic epithelium from people at high risk for colon cancer are complex, there is evidence to suggest that hyperproliferation precedes the development of neoplasia, at least in some instances [1-3,5].

The appearance of neoplasia in the colorectum is associated with a wide range of phenotypic changes. Prominent among these is the increased expression of a variety of tumor-associated glycoproteins. Tumor markers are first found in benign neoplastic colonic lesions, i.e., adenomatous polyps, and increase in expression as the lesions grow and degenerate into cancers [6]. These would appear to be suitable intermediate biomarkers; however, as a group, they tend to occur relatively late in the neoplastic sequence.

\section{GLYCOCONJUGATE MARKERS OF BIOLOGICAL ACTIVITY IN THE COLON}

Lectins are naturally-occurring agglutinins, most of which are derived from plant seeds, that may be used to identify or purify specific glycoconjugate structures. Lectins have been used for over ten years as histochemical probes in the colon, and some have been developed to help identify each of the above mentioned biological activities.

\section{Lectins As Proliferation Markers}

The lectin Amaranthus caudatus agglutinin (ACA) is a recently characterized lectin that labels cytoplasmic glycoconjugates at the base of the normal human colonic crypt. ACA recognizes the T-antigen (Gal $\beta 1,3$ GalNAc $\alpha-O$ ser/thr) or its sialylated forms (NeuAc $\alpha 2,3$ Gal 1,3GalNAc or NeuAc $\alpha 2,3$ Gal1,3 (NeuAc $\alpha 2,6\}$ GalNAc) [7]. Lectin histochemistry was performed using formalinfixed, paraffin-embedded tissue sections that were hydrated, incubated with a biotinylated lectin, and developed with peroxidase conjugated streptavidin [8]. Labeling is greatly increased in adenomatous polyps and cancers, where there is no compartmentalization of labeling as seen in the normal colon [8].

A study was undertaken to compare $\mathrm{ACA}$ labeling (using biotinylated lectin and avidinbiotin-peroxidase to localize lectin binding) with BrdU incorporation in freshly obtained colonic tissues from patients with negative family histories for colorectal cancer who had just undergone a colonoscopy that revealed no 
neoplastic lesions [9]. After $4 \mathrm{hrs}$ of incubation with BrdU, approximately $10 \%$ of cells were labeled in each colonic crypt, the labeling was compartmentalized in the lower two thirds of the crypt, and there was no difference in labeling index between biopsies taken from proximal or distal colons. In adenomatous polyps over $20 \%$ of cells were labeled, and approximately $40 \%$ of cells were labeled in adenocarcinomas using the same technique. Using ACA histochemistry, labeling was seen in the lower two thirds of the crypt in $96.1 \pm$ $2.4 \%$ of colonic crypts and in $2.4 \pm 1.3 \%$ of the upper third of crypts in the distal human colon. However, in the proximal colon, labeling was seen in the lower two thirds of $87.9 \pm 4.6 \%$ of crypts and in the upper one third in $21.2 \pm 3.7 \%$. Thus, ACA was a reliable marker of the proliferative region only in the distal colon. ACA labeled $76 \%$ of glands in adenomatous polyps and $93 \%$ of glands in cancers. Those lesions that showed increased proliferation and loss of compartmentalization using BrdU showed similar changes using ACA. BrdU (and 3H-thymidine) label individual cells that are synthesizing DNA. ACA is somewhat different, and labels contiguous groups of cells in the proliferative region. The precise molecular species labeled by ACA at the base of the colonic crypt is still unidentified.

ACA was used to examine rectal biopsies of normal-appearing tissues of patients with Familial Adenomatous Polyposis (FAP) and hereditary nonpolyposis colon cancer (HNPCC). Since many of these tissues were obtained by colonoscopic biopsy and were not oriented such that the entire crypt could be evaluated in each instance, a biometric scoring system was devised whereby each crypt was scored from 0-4+ depending upon the intensity of labeling. By counting 100 crypts, a score was developed for each biopsy that ranged from 0 (i.e., no labeling in any crypt) to 400 (i.e., 4+ labeling in all 100 crypts).

The average score in a group of normal rectal biopsies was $65 \pm 33$. The labeling score in HNPCC was similar $(74 \pm 70)$, but included five outliers that had an average labeling score of $203 \pm 43$, which was similar to the average scores in FAP $(224 \pm 76)$. Thus, excess labeling with ACA was seen in FAP, compatible with its known hyperproliferative characteristics, and a small subset of patients with HNPCC appeared to have the same abnormality [8]. Although many questions remain to be addressed with ACA, it is a candidate marker for the proliferative pool of cells in the distal normal human colon and in neoplastic lesions.

\section{Lectins As Differentiation Markers}

As noted above, markers of cellular differentiation in the human colon have been less well explored than proliferation markers. However, a series of lectins has been developed that serve this function [4]. The lectins Dolichos biflorus agglutinin (DBA) and soybean agglutinin (SBA) label goblet cell mucin in the normal colon with progressively increasing intensity moving from the base to the surface of the crypt. Contrariwise, the lectins Ricinus communis agglutinin 1 (RCA1) and Bauhinia purpurea agglutinin (BPA) label goblet cell mucin most intensely in the lower portion of the crypt and the intensity decreases progressively as the cells migrate towards the lumen. DBA and SBA label terminal $\alpha-N$-acetylgalactosamine (GalNAc) structures whereas $\mathrm{RCA}_{1}$ and BPA label terminal $\beta$-galactose ( $\beta$-Gal) residues. Based upon the careful structural work of Podolsky on oligosaccharides purified from human colonic mucin $[10,11]$, a reasonable interpretation is that immature sugar side chains are produced in the lower portion of the crypt, and as the cells differentiate and migrate to the surface, a terminal $\alpha$-GalNAc residue is added to the immature chain. However, this speculation has not yet been confirmed.

Using global assessment, a reduction in DBA labeling has been found in mucins in colon cancers, adenomatous polyps, and rectal biopsies from patients with chronic ulcerative colitis. Using the more rigorous biometric described above (resulting in scores from 0-400) a significant reduction in DBA labeling was found in microscopic polyps of patients with FAP. Of interest, significant reductions were also seen in rectal biopsies obtained from patients at risk for HNPCC [12]. With this background, it would be interesting to determine whether lectin markers of differentiation can be used as intermediate biomarkers to assign neoplastic risk to colonic tissues or to assess the impact of chemopreventive programs in patients who are at high risk for cancer. 


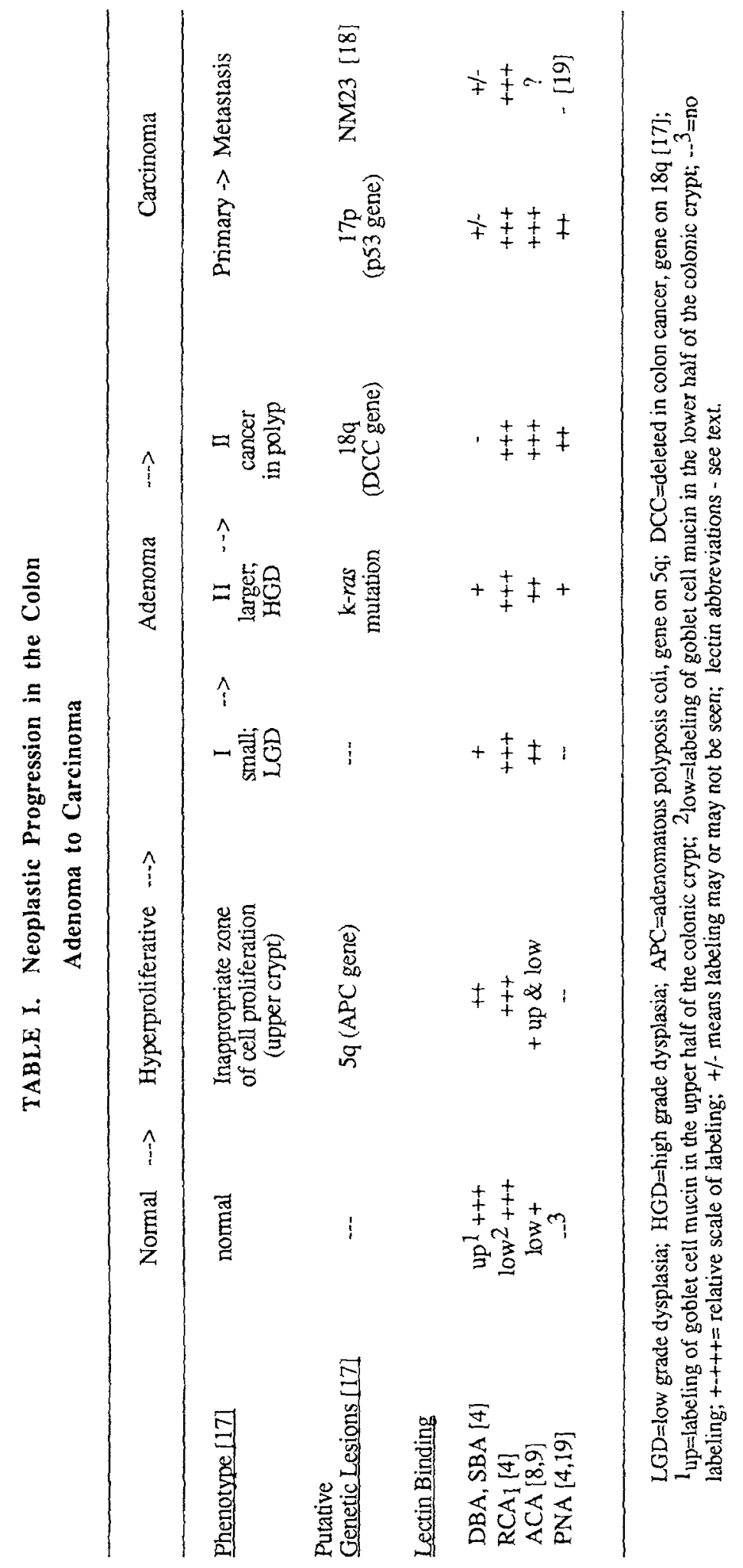


TABLE II. Neoplastic Progression in Ulcerative Colitis

Colitis -.. Dysplasia -.- Carcinoma

\begin{tabular}{|c|c|c|c|c|c|c|}
\hline & Normal $\rightarrow$ & Hyp & erproliferative $\quad-->$ & \multicolumn{2}{|c|}{ Dysplasia } & Carcinoma \\
\hline Phenotype & $\mathrm{NL}^{1}$ & Repair & $-->$ Indeterminate ?--> & LGD ?--> & HGD $\quad-->>$ & Cancer \\
\hline \multicolumn{7}{|l|}{$\begin{array}{l}\text { Putative } \\
\text { Genetic Lesions }\end{array}$} \\
\hline $\begin{array}{l}\text { aneuploidy [20] } \\
\text { ras mutations [21] }\end{array}$ & - & $\begin{array}{c}?+ \\
-\end{array}$ & $\begin{array}{l}?+ \\
-\end{array}$ & $\begin{array}{l}+ \\
-\end{array}$ & $\begin{array}{l}+ \\
+\end{array}$ & $\begin{array}{l}+ \\
+\end{array}$ \\
\hline \multicolumn{7}{|l|}{$\begin{array}{l}\text { Lectin } \\
\text { Binding [22] }\end{array}$} \\
\hline $\begin{array}{l}\text { DBA } \\
\text { PNA }\end{array}$ & $\begin{array}{c}\text { NL }(+++) \\
-\end{array}$ & $\stackrel{++}{\text { Golgi }} 2$ & $\begin{array}{l}++ \\
\text { Golgi }\end{array}$ & $\begin{array}{l}++ \\
\text { Golgi }\end{array}$ & $\begin{array}{c}++ \\
+ \\
\text { (mucin) }\end{array}$ & $\begin{array}{c}+/-[4] \\
+ \\
\text { (mucin) }\end{array}$ \\
\hline
\end{tabular}

${ }^{1} \mathrm{NL}=$ same as seen in normal colonic tissues (see Table 1); LGD=low grade dysplasia; HGD=high grade dysplasia; ${ }^{2}$ Golgi=faint labeling seen but limited to the supranuclear region and presumed to represent the Golgi apparatus; lectin abbreviations - see text.

\section{Lectins As Tumor Markers}

Lectin markers of malignant transformation have also been developed. Peanut agglutinin (PNA) binds to the T-antigen, which is expressed in the mucin secreted by most colorectal cancers [4]. Of interest, this lectin binds to adenomatous polyps in a stepwise fashion that correlates with other indicators of malignant risk in the polyp such as size, villous content, and the degree of dysplasia [6]. PNA has been found to be a marker of early neoplastic change in the colons of CF-1 mice treated with carcinogen [13], and in a marmoset model of spontaneous colitis and colon cancer [14]. Of interest, PNA is a differentiation marker in the proximal colon of CF-1 mice, where it selectively binds goblet cell mucin in the lower half of the colonic crypt [13]. By contrast, PNA does not bind anywhere in the normal human colonic crypt. PNA is not a specific marker for colon cancer, since faint degrees of labeling can be seen in inflamed colons [15], and moreover, labeling is found in hyperplastic polyps of the colon, which do not behave as neoplasms [6]. PNA binds to glycoconjugates of first trimester human fetal colon, making it an oncofetal marker [16].

\section{GENETIC CONTROL OF MARKER EXPRESSION}

The sequential changes in glycoconjugate expression in the adenoma-to-carcinoma sequence are outlined in Table 1 , and correlated with putative sequential changes that occur at the genetic level during the genesis of a neoplasm [17-22]. It is tempting to speculate a possible cause and effect relationship between the genetic changes and the altered expression of mucin structure.

A similar, highly speculative correlation can be drawn between the abnormal expression of mucin-type glycoproteins in ulcerative colitis in the putative dysplasia-tocancer sequence in that disease (Table 2). Of interest, the appearance of glycoconjugates that are bound by PNA appear relatively late in both schemes, at approximately the time that the ras gene mutation is thought to appear $[17,19,21,22]$. The genetic events 
responsible for the selective expression of glycoproteins in colonic tissues of different biological behavior remains to be explored.

\section{SUMMARY AND CONCLUSIONS}

Lectins have been found that serve as markers of proliferation, differentiation, and malignant transformation. Lectin binding studies can be performed on fixed, paraffinembedded tissues, including archival tissues that have been stored for prolonged periods. Lectins may be conjugated with fluorescein for fluorescence microscopy or with biotin for peroxidase labeling that utilizes light microscopy. Tissue biometrics have been developed to score the labeling, which permits a quantitative assessment of partial changes in tissue expression of glycoconjugates. Early events in the colorectal neoplasia progression sequence include increases in proliferation and a partial failure of complete cellular differentiation. These may be assayed using the lectins ACA, DBA, and SBA. Later events involve the total loss of expression of differentiation markers and the appearance of the tumor antigen recognized by PNA. The clinical utility of these markers in identifying patients at high risk for colon cancer or for measuring the impact of chemopreventative agents on the colon remains to be tested. Depending on the needs of the study, a panel of lectins may be selected, histochemical scores may be quantitated by observers blinded to the therapy, and these studies may be repeated serially over time. The limitation of this approach is that an invasive procedure will be required to obtain tissue for labeling. The use of colonic effluent or fecal material for these studies might be unsatisfactory because of the abundant presence in the colonic lumen of enzymes that degrade glycoprotein structures. In spite of these shortcomings, lectins appear to be among the most promising probes to be developed as intermediate biomarkers of neoplasia in the colon.

\section{REFERENCES}

1. Lipkin M, Higgins P: Biological markers of cell proliferation and differentiation in human gastrointestinal diseases. Adv Cancer Res 50:124,1988 .

2. Risio M, Lipkin M, Candelaresi G, Bertone A, Coverlizza S, Rossini FP: Correlations between rectal mucosa cell proliferation and the clinical and pathological features of nonfamilial neoplasia of the large intestine. Cancer Res 51:19171921, 1991.

3. Luk GD, Baylin SB: Ornithine decarboxylase as a biologic marker in familial colonic polyposis. N Engl J Med 311:80-83, 1984.

4. Boland CR, Montgomery CK, Kim YS: Alterations in human colonic mucin occurring with cellular differentiation and malignant transformation. Proc Natl Acad Sci, USA 79:20511055, 1982.

5. Deschner EE, Lipkin M, Solomon C: Study of human rectal epithelial cells in vitro. II. ${ }^{3} \mathrm{H}$. thymidine incorporation into polyps and adjacent mucosa. J Natl Cancer Inst 36:849-857, 1966.

6. Boland CR, Montgomery CK, Kim YS: Cancerassociated mucin alterations in benign colonic polyps. Gastroenterology 82:664-672, 1982.

7. Rinderle SJ, Goldstein IJ, Matta KL, Ratcliffe RM: Isolation and characterization of amaranthin, a lectin present in the seeds of Amaranthus caudatus, that recognizes the $\mathrm{T}$-(or cryptic T)-antigen. J Biol Chem 264:16123-16131, 1989.

8. Boland CR, Chen Y-F, Rinderle SJ, Resau JH, Luk GD, Lynch HT, and Goldstein IJ: The use of the lectin from Amaranthus caudatus as a histochemical probe of proliferating colonic epithelial cells. Cancer Res 51:657-665, 1991.

9. Martin MA, Poore JA, Kraus ER, Boland CR: Assessment of colonic tissue proliferation using bromodeoxyuridine (BrdU) incorporation and amaranthin (ACA) histochemistry. Gastroenterology 100:A383, 1991.

10. Podolsky DK: Oligosaccharide structures of human colonic mucin. J Biol Chem 210:8262-8271, 1985.

11. Podolsky DK: Oligosaccharide structures of isolated human colonic mucin species. J Biol Chem 260:15510-15515, 1985.

12. Sams JS, Lynch HT, Burt RW, Lanspa S, Boland CR: Abnormalities of lectin histochemistry in familial polyposis coli and hereditary nonpolyposis colorectal cancer. Cancer 66:502-508, 1991.

13. Boland CR, Ahnen DJ: The binding of lectins to goblet cell mucin in premalignant colonic epithelium of the CF-1 mouse. Gastroenterology 89:127$137,1985$.

14. Boland CR, Clapp NK: Glycoconjugates in the colons of new world monkeys with spontaneous colitis and cancer: the association between inflammation and neoplasia. Gastroenterology 92:625-634, 1987.

15. Lance P, Boland CR: Abnormal goblet cell mucin in non-neoplastic colonic disease. Clin Res 32:282A, 1984.

16. Boland CR, Kim YS: Peanut lectin binding identifies human colonic onco-fetal mucin. Clin Res 30:34A, 1982.

17. Fearon ER, Vogelstein B: A genetic model for colorectal tumorigenesis. Cell 61:759-767, 1990.

18. Cohn KH, Wang F, DeSoto-LaPaix F, Solomon WB, Patterson LG, Arnold MR, Weimar J, Feldman JG, Levy AT, Leone A, Steeg PS: Association of $n \mathrm{~m} 23-\mathrm{H} 1$ allelic deletions with 
distant metastases in colorectal carcinoma. Lancet 338:722-724, 1991.

19. Bresalier RS, Boland CR, Kim YS: Characteristics of colorectal cancer cells with high metastatic potential. Gastroenterology 87:115-122, 1984.

20. Hammarberg C, Slezak P, Tribukait B: Early detection of malignancy in ulcerative colitis. A flow-cytometric DNA study. Cancer 53:291-295, 1984.

21. Meltzer SJ, Mane SM, Wood PK, Resau JH,
Newkirk C, Terzakis JA, Korelitz BI, Weinstein WM, Needleman SW: Activation of c-Ki-ras in human gastrointestinal dysplasias determined by direct sequencing of polymerase chain reaction products. Cancer Res 50:3627-3630, 1990.

22. Boland CR, Lance P, Levin B, Riddell RH, Kim YS: Abnormal goblet cell glycoconjugates in rectal biopsies associated with an increased risk of neoplasia in patients with ulcerative colitis: Early results of prospective study. Gut 25:13641371 . 